\title{
Coupling charge blasting research
}

\author{
Xie Huagang1, a Wu Lingli1,b Fan Yanqii, ${ }^{2, c}$ Wang Guoguo ${ }^{3, d} \quad$ Pan Jun ${ }^{1, e}$ \\ Cheng Kun ${ }^{1, f}$
}

\author{
1.Tongling University, Tongling 244061, Anhui Province, China \\ 2.Jiangsu Institute of Water Resources Institute of Materials Structure, Yangzhou 225100, \\ Jiangsu Province, China
}

3.Shandong Provincial Communications Planning and Design Institute, Ji'nan 250031, Shandong Province, China

aemail:xiehg2005@126.com, bemail: 181531464@qq.com, cemail: 249891473@qq.com, demail: 254065360@qq.com, eemail: 278203271@qq.com, ${ }^{\mathrm{f} e m a i l: 372575185 @ q q . c o m}$

Keywords: Coupling charge; Theoretical research; Numerical simulation; Field application

Abstract: From the theory of coupled charge blasting, the coupling charge blasting test and the field application of coupled charge blasting, the total energy of coupled charge explosive burst stress wave is the strongest, and the explosive energy is more used to crush the near borehole medium .

\section{Introduction}

With the widespread application of controlled blasting technology, many projects involve the control of high-water-content controlled blasting, such as smooth blasting, presplitting blasting and controlled blasting controlled blasting in the construction of aquifers and underwater wharf in roadway engineering. However, the physical and mechanical properties of the surrounding rock of the roadway essentially determine the blasting effect. At the same time, the degree of coupling between the explosive and the surrounding medium is also one of the key factors that affect the blasting effect. The uncoupling or the poor coupling will make the rock The intensity of the explosive stress wave excited by explosives in the body decreases ${ }^{[1]}$ 。 In order to reduce the impact of rock excavation blasting on the bearing rock mass damage and vibration, the contour blasting technique such as presplitting or smooth blasting is usually adopted in the rock excavation profile. Conventional contouring often use uncoupled charge structure, such as the emulsion explosives lashing in the hole throughout the detonating cord to achieve radial and axial charge coupling to control the blast hole blasting pressure and blasting damage effect ${ }^{[2]}$ 。 Traditional construction, in order to ensure the uncoupling coefficient, most of the use of large-caliber DTH drilling holes, Ф32 standard drug interval interval charge. If you need to use a hand drill, you must make a special roll diameter, increase production costs. Coupling charge structure, free to use drilling tools, drug roll diameter is not limited ${ }^{[3]}$ 。

\section{Coupled charge theory}

Blasting explosives in the rock mass produces shock waves, stress waves and elastic seismic waves. According to the measured data, in hard rock, the shock wave propagates within a short distance around the explosive core, and the shock wave pressure is extremely high, which is far 
greater than the dynamic compressive strength of the rock, so that the rock can be crushed but the propagation distance is short and the crushing range is small, The area corresponding to the shock wave is called the crushing zone. In order to obtain the calculation of the radius of smashing circle, Jiangdong et al. Calculated the explosive detonation velocity based on the characteristics of mass charge, momentum conservation, energy conservation and the equation of state, H-peak pressure. Reference to previous studies, detonation wave geometric decline. Through the analysis of the stress intensity of the smashing ring particles and the limit failure conditions of the crushing zone, the radius of the smashing ring is obtained. Using the obtained formula, the crushing zone radius rf and the hole radius rb ratio of some common rock masses were calculated. Compared with Hanukayev's research, the calculated values basically corresponded ${ }^{[4]}$ 。 Expansion Blasting is widely used in the construction of mines, highways, railways, various power plants and military installations. However, in the expansion blasting, engineering designers mainly rely on experience in design and construction, prone to accidents; coupled with its theoretical research has lagged behind the actual needs, and because of the limitations of experimental methods and related theories are still not perfect Exploring stage. In order to get the theoretical formula of the radius of expansion in coupling charge, Wu Jianxing et al. Established the physical model of expansion blasting according to the expansion mechanism and obtained the radius formula of the final blast hole extrusion area under different conditions according to the law of conservation of momentum. The use of these formulas combined with the characteristics of explosives and rocks, as well as the dosing parameters have been dilated. The theoretical calculation results are consistent with the experimental data. These results will help to improve the theory of blasting capacity expansion and the development and application of the technology ${ }^{[5]}$ 。

\section{Coupled charge blasting experimental study}

$\mathrm{Wu}$ Liang and others ${ }^{[2]}$ used dynamic finite element software LS-DYNA to analyze the failure mechanism of air-gap charge and coupling charge structure on concrete medium, and carried out smooth blasting test on two kinds of charge structures with concrete model. The peak pressure of the cell wall of the coupled charge structure is about $3.8 \mathrm{GPa}$, while the pressure peak value of the cell wall of the air-gap charge structure is $2.0 \mathrm{GPa}$. Therefore, the explosive energy of the coupled charge is more used for crushing the near bore medium, Resulting in smooth surface near blast hole when the medium surface roughness is poor. The blasting effect is shown in Fig.1(a) and Fig.1(b). It can be seen from Fig.1(a) and Fig. 1(b) that both blasting methods can achieve good blasting effect. The air space charge structure, the presence of air layer reduces the peak pressure of blast hole and prolongs the blasting action time, reduces or even avoids the impact crushing of ore rock formed by the coupling charge, expands the scope of the rupture zone, increases the explosive Effective utilization of explosive energy. In addition, the air gap charge can adjust the energy distribution of explosives, so that the hole explosion pressure distribution along the borehole axis more evenly, so using air space charge structure blasting, the concrete surface roughness is better than the coupling charge structure After the blasting situation. Xu Guangbin ${ }^{[6]}$ combined LS-DYNA3D program, introduced the dynamic finite element method of blasting numerical simulation theory, and the use of procedures to make two adjacent hole coupling charge bench-scale deep hole blasting model example. Compared with the engineering practice, the calculation result is reasonable and the simulation method is feasible. The results show that the numerical simulation can get a more complete description of the motion, the time-history analysis of the effective blasting stress and the visual demonstration of the propagation of the blasting stress wave. 


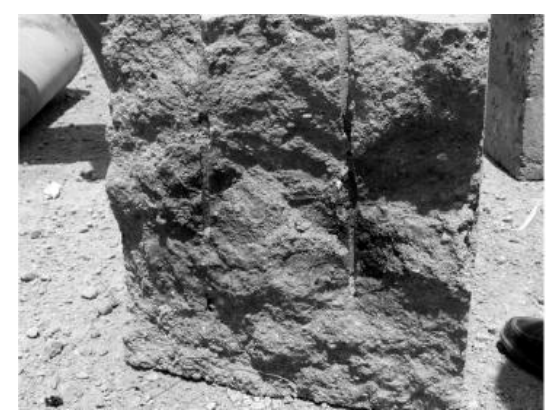

a

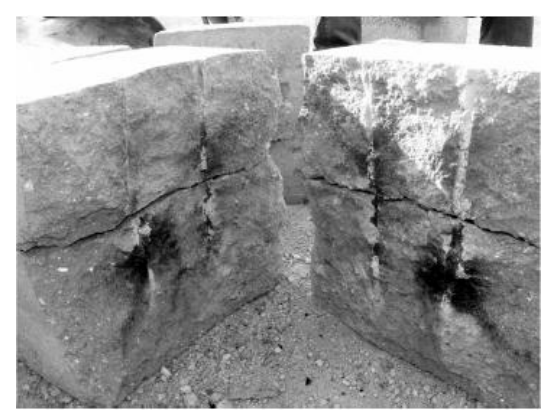

b

Fig. 1 Blasting effect ${ }^{[2]}$

a axial coupling charge structure b、 air-deck charge structure

\section{Coupled charge blasting field applications}

Cai Feng and others ${ }^{[7,8]}$ on the charge coefficient at Huainan Mining Group Pansan Mine 1311 (1) and 1303 (1) wall surface air back groove study. The burial depth of coal seam is $690 \mathrm{~m}$ and $706 \mathrm{~m}$, and the gas content is $9.3 \mathrm{~m} 3 / \mathrm{t}$. The elastic modulus of coal seam where the experiment site is located is $1.5 \mathrm{Gpa}$ and $1.4 \mathrm{Gpa}$. Blasting hole construction drill diameter $\Phi 75 \mathrm{~mm}$, drilling depth of $8 \mathrm{~m}$ and $7 \mathrm{~m}$, blast hole filled with deep hole pre-cracking blasting special drug column, the gap between the column and the explosion hole wall air, the charge length of $1 \mathrm{~m}$, Sealing length of $7 \mathrm{~m}$, the use of deep-hole presplit blasting special sealing equipment and sealing materials sealing hole, you can effectively resist the impact of detonation to prevent punching. The geophones are arranged $3 \mathrm{~m}$ away from the center of the explosion hole. The distance between the geophones is $3 \mathrm{~m}$. A total of 40 channels are provided. The last geophone is $120 \mathrm{~m}$ away from the explosion hole. Charge structure and detector layout shown in Fig.2. Finally, the field experiments on the influence of the stress wave energy by the coupling charge coefficient were carried out. When the coupling charge coefficient $\xi=1.5$, the low frequency energy of the burst stress wave signal is the strongest and the signal-to-noise ratio is the highest in the range of $10 \sim 50 \mathrm{~Hz}$. At the same time, the theoretical derivation shows that although the total energy of the burst stress wave is the strongest when the coupling charge coefficient $\xi=1.0$, the main frequency of the burst stress wave with the coupling charge coefficient $\xi=1.5$ is found by theoretical analysis and numerical calculation. Lower, more energy and high signal-to-noise ratio, can effectively improve the effect of explosive stress wave excitation. The total energy of a burst stress wave can be made up by increasing the charge. $\mathrm{He}$ Jinsong $^{[3]}$ exploited coupling charge to excavate the rock at the entrance of Yili Shankou Power Station and the sidewalls of the Drainage Channel in 1996. The blasting method is step blasting. The coupling charge structure is adopted, and the pre-cracking blasting is successful. The half-porosity rate is $>80 \%$. 1998 In the case of excavation and blasting of the secondary dam retaining wall of Shihmenzi Reservoir in the trench excavation and blasting, the coupled charge structure is adopted, and the presplitting blasting is successful with the half porosity of $>80 \%$. In addition, the right abutment cutting slope, which is conducive to hand-drilling holes, the use of coupling charge smooth blasting, blasting has also been a success. Therefore, it is considered that in the case of stepped blasting, coupling charge smooth blasting can also be used to make a seam. 


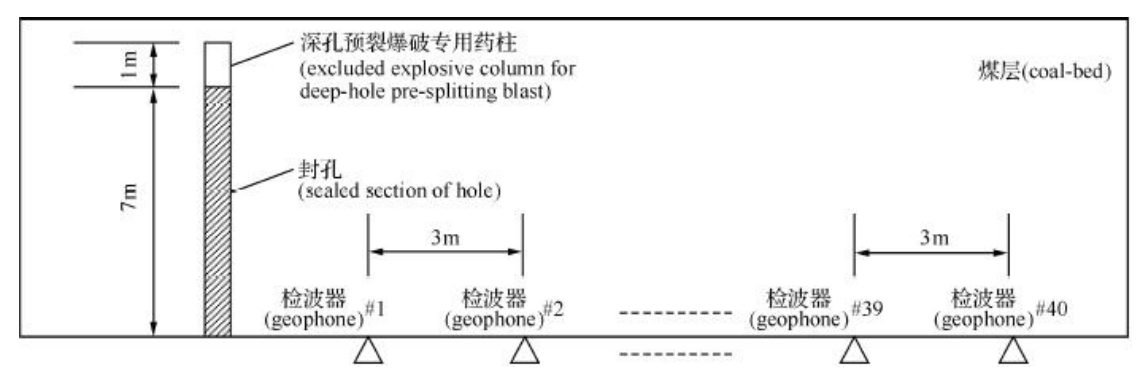

Fig. 2 The diagram of charge structure and sensors ${ }^{[7]}$

\section{Conclusions}

Although some achievements have been made in the research on coupling charge blasting at home and abroad, it has been widely used in engineering applications and has been affirmed by researchers. However, due to the complicated mechanism of explosion, there are still many problems worthy of our study To study. The following is the conclusion of the existing achievements of coupling charge blasting and some suggestions for future research directions:

1. Coupled charge after blasting Explosive stress wave of the strongest total energy, at the same time more explosive energy used to smash the borehole near the media;

2 . In the aspect of numerical simulation, meshless method has obvious advantages over the finite element in the problems of grid distortion and grid movement, and is more conducive to simulate the explosion process of coupled charge blasting. At present, there are few numerical simulation studies in this area and further research is needed.

\section{Acknowledgements}

This work was financially supported by the 2017 Anhui Provincial Outstanding Young Talent Support Program(gxyq2017080); 2017 Anhui Provincial College of Natural Science Research Key (Major) Project (KJ2017A473); 2016 Anhui provincial quality project (2016ckjh214) ; 2016Provincial College Students Innovation and Entrepreneurship Training Program (201610383069); 2016 Provincial College Students Innovation and Entrepreneurship Training Program Project (201610383074); 2017 National College Students Innovation and Entrepreneurship Training program Project (201710383027); 2017 National College Students Innovation and Entrepreneurship Training Project (201710383028)

\section{References}

[1] GAO Bao-bin, LIU Yun-peng, WANG Zu-guang. Analysis of blasting effect under different coupling charge structure[J]. Journal of Hunan University of Science \& Technology( Natural Science Edition), 2014, (03): 10-14.

[2] WU Liang, ZHOU Yong, YANG Dan, et al. Contrastive analysis of air-deck charge and coupling charge in smooth blasting[J]. Journal of Wuhan University of Science and Technology, 2012, (03): 225-228.

[3] HE Jin-song. Application of large volume smooth blasting technology in cutting slope excavation[J]. Xinjiang Water Resources, 2006, (05): 39-40.

[4] JIANG Dong-ping, ZHU Lei. Calculation of radius of blasting powdering region under coupling charge condition[J]. Morden Mining, 2013, (04): 14-15+18.

[5] WU Jian-xing, ZHANG Jian-hua. Theoretical calculation of springing chamber radius in coupling charge[J]. Industrial SAF ETY and Environmental Protection, 2007, (09): 47-49. 
[6] XU Guang-bin. Numerical simulation of deep_hole bench blasting with coupling charge[J]. GX Water Resources \&Hydropower Engineering, 2007, (03): 20-23.

[7] CAI Feng, LIU Ze-gong. Analytic method of earthquake action calculation for multistory isolated structure[J]. Chinese Journal O F Applied Mechanics, 2014, (03): 332-337+48.

[8] CAI Feng, LIU Ze-gong. Impact of coupling charge properties on energy attenuation of stress waves of deep-hole presplit blast[J]. Journal of China Coal Society, 2014, (S2): 384-389. 